\title{
Amniotic Membrane Application Reduces Liver Fibrosis in a Bile Duct Ligation Rat Model
}

\author{
Luciana B. Sant'Anna,* Anna Cargnoni,* Lorenzo Ressel,* Graziella Vanosi, $†$ and Ornella Parolini* \\ *Centro di Ricerca E. Menni, Fondazione Poliambulanza-Istituto Ospedaliero, Brescia, Italy \\ $\nmid$ Dipartimento di Scienze Cliniche Veterinarie-Sezione di Radiologia Clinica e Sperimentale, Facoltà di Medicina Veterinaria, \\ Università degli Studi di Milano, Milano, Italy
}

\begin{abstract}
Biliary fibrosis and resultant cirrhosis are among the most common outcomes of chronic liver diseases. Currently, liver transplantation remains the only effective treatment. In seeking alternative therapeutic approaches, we focused on the potential use of the human amniotic membrane (AM). Indeed, AM has gained increasing importance for its antiscarring, anti-inflammatory, and wound-healing properties, as well as for the multipotent differentiation ability and immunomodulatory features of AM-derived cells. Intriguingly, we have recently demonstrated that placenta-derived cells reduce lung fibrosis in bleomycin-treated mice, and that AM patches reduce postischemic cardiac injury in rats. Hence, we have now investigated the effects of human AM on biliary fibrosis induced in rats through the bile duct ligation (BDL) procedure. A fragment of human AM was applied onto the liver surface after BDL and the effects on fibrosis establishment and progression were evaluated at different time points in comparison with fibrosis progression in control BDL rats. The degree of liver fibrosis was first assessed by the semiquantitative Knodell scoring system and, thereafter, by digital image morphometric analysis to quantify the area occupied by ductular reaction, activated myofibroblasts, and collagen deposition. We demonstrated a significant reduction in the severity of BDL-induced fibrosis in AM-treated rats. Indeed, while fibrosis progressed rapidly in control BDL rats, leading to cirrhosis within 6 weeks, AM-treated rats showed confined fibrosis at the portal/periportal area with no signs of cirrhosis, and a reduction in collagen deposition to about $50 \%$ of levels observed in control BDL rats. In addition, the AM was able to significantly slow the gradual progression of the ductular reaction and reduce, at all time points, the area occupied by activated myofibroblasts. These findings suggest that human AM, when applied as a patch onto the liver surface, might inhibit fibrosis progression in BDL-injured livers, and could protect against hepatic damage associated with fibrotic degeneration.
\end{abstract}

Key words: Human amniotic membrane; Placenta cells; Bile duct ligation; Liver fibrosis; Ductular reaction; Myofibroblasts

\section{INTRODUCTION}

Liver fibrosis is a common outcome of a variety of chronic liver diseases following different insults, such as viral infection, alcoholism, chemical toxicity, or metabolic and biliary disorders $(23,38)$. Following repeated injuries, the liver undergoes fibrotic remodeling characterized by an excessive accumulation of extracellular matrix (ECM) in the liver parenchyma that distorts the normal hepatic architecture, with the formation of scar tissue encapsulating the area of injury (11). Progressive fibrosis could end in the advanced stage known as cirrhosis, characterized by fibrotic septa and rings of scar tissue that surround regenerative nodules of liver parenchyma $(11,38)$. Patients with cirrhosis have a high risk of developing irreversible liver failure or hepatocellular carcinoma in later life (46).

Despite decades of research that has led to substantial advances in understanding the molecular mechanisms of this disease's progression, hepatic cirrhosis remains a pathology that is difficult to treat in an effective manner unless orthotopic liver transplantation is performed $(31,43)$. However, this approach presents with several limitations of its own, such as: i) scarce availability of donor livers, ii) the need to commit transplant patients to life-long toxic immunosuppression; iii) the fact that many patients may be unsuitable for transplantation (35). Therefore, alternative therapies are urgently needed.

More recently, hepatocyte transplantation has become a promising therapeutic tool for a variety of liver dis- 
eases $(19,20,51)$. However, this approach is also currently limited by different factors, such as the restricted availability of liver for hepatocyte isolation, the poor quality of the cells obtained after the isolation procedure, the modest level of engraftment achieved in the host liver after transplantation, and low cell viability, which is one of the reasons that long-term beneficial effects are often not observed following hepatocyte transplantation $(19,20,32)$.

The use of stem or progenitor cell transplantation is emerging as a potential alternative to hepatocyte transplantation for the treatment of liver disorders $(36,44)$, and several potential sources have been identified for the isolation of these cells (28). For the treatment of liver fibrosis, this approach has been performed mainly using bone marrow-derived mesenchymal stromal cells (MSCs), although the results that have been obtained so far are controversial (reduction vs. enhancement of fibrosis), and open questions also remain regarding the mechanisms involved $(1,11)$. At least two possible mechanisms have been proposed for the potential therapeutic function on liver fibrosis exerted by MSCs: one implies their ability to engraft into the liver and differentiate towards the hepatogenic lineage, therefore participating in the regeneration of the endogenous parenchyma; the other possible mechanism, which seems the most likely, is related to the ability of MSCs to produce/ activate paracrine mediators, such as IL-10, TNF- $\alpha$, and $\mathrm{HGF}$, which lead to reduction of fibrosis [for a review see Dai et al. (11) and Henderson and Forbes (23)].

Many issues remain to be addressed before stem cell therapy can be considered as a viable clinical strategy for such a liver disease, and among these is the need to identify a source of stem cells that is safe, does not pose any risk of tumor induction and/or risk of viral infection (16), is easily accessible, provides a high cell yield, and for which cell procurement does not provoke ethical debate $(17,32)$. To this end, the human amniotic membrane (AM) represents a prime candidate as a possible source of stem/progenitor cells. Cells isolated from this membrane have a stem cell phenotype: human amniotic epithelial cells (hAEC) express pluripotent stem cell markers and are able to differentiate in vitro toward tissues of all three germ layers $(33,37,53)$, while human amniotic mesenchymal stromal cells (hAMSC), like MSCs of other origins, have the capacity to differentiate towards cells of the mesodermal lineages, as well as toward tissues of ectodermal and endodermal layers $(39,49,53)$.

Several findings suggest that human AM-derived cells might be useful for restoring the functionality of hepatic tissues that have been compromised by disease or injury.

On one hand, there are data suggesting the potential ability of human AM-derived cells to engraft in the liver
(4) and also to differentiate toward the hepatogenic lineage in vitro and in vivo $(34,52,54)$.

On the other hand, several studies testify that transplantation of an intact AM fragment exerts anti-infammatory and antiscarring effects reviewed in Gomes et al. (22), Fernandes et al. (18), and Dua et al. (15). In addition, more recently, preclinical studies have shown that, likely by means of paracrine mechanisms, transplantation of xenogenic and allogenenic placenta-derived cells reduces lung fibrosis in bleomycin-treated mice (9), while application of a human AM patch onto ischemic rat hearts results in improved cardiac function (8). Taken together, these findings suggest that transplantation of AM-derived cells and/or AM itself could be a novel therapeutic strategy to treat hepatic diseases.

The purpose of this study was to evaluate the effects of human AM patches on the establishment and progression of biliary-type liver fibrosis induced in rats through the bile duct ligation (BDL) procedure.

\section{MATERIALS AND METHODS}

\section{Animals and Experimental Groups}

All animal experiments were carried out in accordance with current Italian and European regulations and laws on the Use and Care of Animals for research (DL. 116/27 January 1992). Sixty female Wistar rats (Laboratoire Elevage Janvier, France), weighing 220-250 g, were housed at controlled temperature $\left(22 \pm 2{ }^{\circ} \mathrm{C}\right)$ with daily exposure to a 12:12 light/dark cycle and allowed unlimited access to chow and water. Before any experimental intervention, the rats were randomly divided into two experimental groups of 30 rats each, as follows: BDL group - rats subjected to the BDL procedure and euthanized at $2(n=10), 4(n=10)$, and 6 weeks $(n=10)$ after ligation; BDL + AM group-rats subjected to the BDL procedure and treated with human AM and euthanized at $2(n=10), 4(n=10)$, and $6(n=10)$ weeks after ligation.

\section{Human AM Collection}

Human term placentas were obtained from caesarean sections or spontaneous deliveries with maternal consent according to the guidelines of the Ethical Committee of the Catholic Hospital (CEIOC). Briefly, the human AM was manually separated from the chorionic membrane and washed extensively in phosphate-buffered saline (PBS) (Sigma, St. Louis, MO, USA) containing 100 U/ $\mathrm{ml}$ penicillin and $100 \mu \mathrm{g} / \mathrm{ml}$ streptomycin (Lonza, Basel, Switzerland). The AM was then cut into pieces of adequate dimension $(6 \times 8 \mathrm{~cm})$ that were stored separately at room temperature, as reported by Hennerbichler et al. (24), in 50-ml vials filled with serum-free and phenol red-free DMEM in sterile conditions, until application. The AM fragments were used within $24 \mathrm{~h}$. As pre- 
viously reported, the number and viability of cells in the human AM fragments stored for $24 \mathrm{~h}$ were not significantly reduced compared to those seen using fresh membrane (8).

\section{Rat Model of BDL-Induced Fibrosis}

The rats were anesthetized with $3 \%$ isoflurane. The abdomen was shaved and disinfected with $10 \%$ povidone iodine and, after a midline abdominal incision, the common bile duct was exposed and ligated by double ligatures with 4-0 suture silk (Johnson \& Johnson Medical S.P.A. Rome, Italy). The first ligature was made below the junction of the hepatic ducts and the second ligature was made above the entrance of the pancreatic duct, as reported by Chen et al. (10). The common bile duct was then sectioned between the ligatures. The abdominal incision was then closed in two layers with 4-0 and 3-0 suture silk. Thus, animals had total, permanent biliary obstruction.

In animals of the AM-treated group, immediately after BDL and before closing of the abdominal wall, an AM fragment was gently placed onto the liver with its mesenchymal side in contact with the liver surface, in such way that the whole surface of the liver lobules was covered. Holding its upper extremities using two forceps, the membrane was inserted under the liver and, with gentle movements, it was then cranially shifted and raised until it covered the surface of the median lobe. The inferior membrane extremities were also raised in order to cover the remaining liver lobes and, finally, the superior and the inferior membrane extremities were attached to each other with a drop of methacrylate glue to keep the membrane in place on the liver, avoiding its dispersion into the peritoneal cavity. After this procedure, the abdomen was closed, as described above for animals of the BDL group. After surgery, each rat was given subcutaneous antibiotic therapy (Marbocyl 1\%, 10 $\mathrm{mg} / \mathrm{kg}$ ) and following the recommendations of Beck and Lee (6), subcutaneous injection of vitamin $\mathrm{K}(5 \mathrm{mg} / \mathrm{kg})$ was administered weekly to decrease mortality from hemorrhagic diathesis.

\section{Liver Explants and Histological Processing}

Two, 4, and 6 weeks after BDL, 10 animals of each group were euthanized with an excess of isoflurane. Liver samples were taken from the major left and median lobes (which comprise approximately $70 \%$ of the liver), fixed in $10 \%$ neutral buffered formalin (for at least $48 \mathrm{~h}$ ), and embedded in paraffin. At least four 4$\mu \mathrm{m}$-thick sections were used from each rat and these were stained with hematoxylin/eosin and with Goldner's modified Masson trichrome staining procedure for fibrosis and collagen analysis, or placed on polysine-L-coated slides (Menzel-Glaser, Braunsch, Germany) for immunohistochemical stainings.

\section{Liver Fibrosis Evaluation}

The degree of liver fibrosis was examined semiquantitatively under a bright field Olympus microscope at $100 \times$ magnification by applying the Knodell fibrosis scoring as follows (7): level 0 , indicating absence of fibrosis; level 1, fibrous portal expansion; level 3, bridging fibrosis (portal-portal or portal-central vein linkage); level 4, cirrhosis. The scoring was performed blindly by a pathologist, who was not aware of the treatments of the rats. The average of the score taken from 10 nonoverlapping random fields per section was used to generate a single score for each specimen.

\section{Immunohistochemistry}

Immunostainings for cytokeratin 19 (CK19) and $\alpha$ smooth muscle actin ( $\alpha$-SMA) were performed to identify bile ductular structures and activated myofibroblasts, respectively. Briefly, each section was immersed into the preheated target retrieval solution (W-CAP citrate buffer pH 6.0) (BioOptica, Milano, Italy) and incubated for $30 \mathrm{~min}$ at $98^{\circ} \mathrm{C}$. This solution achieves, in just one step, deparaffinization and heat-induced epitope retrieval. Endogenous peroxidase activity was blocked with $3 \%$ hydrogen peroxide for $10 \mathrm{~min}$ followed by three washes in PBS containing $0.05 \%$ Tween 20 (Sigma). Sections were then incubated overnight in a humidity chamber at $4^{\circ} \mathrm{C}$ with primary mouse monoclonal antibodies against human $\alpha$-SMA (Clone 1A4, dilution 1:600, Dako Corporation, Denmark), which cross-reacts with the rat antigen $\alpha$-SMA (48), and against CK19 (Clone-b170, dilution 1:100, Novocastra, Newcastle, UK) (45). Sections were again washed, and incubated with secondary antibody using the MACH $4^{\mathrm{TM}}$ Universal HRP-polymer kit (Biocare Medical, Concord, CA, USA) according to the manufacturer's instructions. For visualization, diaminobenzidine (BioGenex Laboratories, San Remon, CA) was applied for $5 \mathrm{~min}$. All sections were counterstained with hematoxylin, dehydrated in alcohol, cleared in xylene, and mounted with Eukitt Mounting Medium ${ }^{\mathrm{TM}}$ (Fluka Biochemika, Steinheim, Germany). Slides were then air dried. The specificity of the antibody-antigen reaction was tested by replacing the primary antibodies with normal serum from the donor species of the first antibody (mouse IgG2a, Dako Corporation, Denmark). As an internal control, vascular smooth muscle and bile duct immunoreactivity were assessed for $\alpha$-SMA and CK19, respectively.

\section{Image Morphometric Analysis}

Goldner's modified Masson trichrome staining, CK19 and $\alpha$-SMA immunostainings were quantitatively evalu- 
ated by digital image analysis. The quantification was performed using the open-source CellProfiler image analysis software (27). The images of all stainings were captured by a digital camera (Olympus Camedia C-4040 ZOOM) and digitalized at $1024 \times 768$ pixel, $24 \mathrm{bit} / \mathrm{pixel}$ resolution with a global magnification of $100 \times$. The digital images were processed by CellProfiler, to identify, isolate, and measure areas occupied by immunostained cells or by collagen deposition over the total liver parenchyma area.

The mean value taken from 10 nonoverlapping random fields per section, all of which contained the central vein and the portal tracts, was used to generate a single value for each specimen.

\section{Nomenclature to Designate Biliary Structures}

The terminology used in this work to designate biliary structures refers to the consensus on nomenclature of the finer branches of the biliary tree published by Roskams et al., and also applied by other authors to describe "ductular reaction" and liver fibrosis after BDL $(5,21,42)$. "Ductular reaction" implies a reaction of ductular phenotype. In the case of injury caused by obstruction of the common bile duct (BDL model), we will use this term to designate the increasing numbers of biliary structures (ductules) (i.e., cholangiocyte proliferation) that arise in response to the injury and that are intensely CK19 positive.

\section{Statistical Analysis}

Data are reported as median and interquartile range (IQR). Kruskal-Wallis nonparametric analysis of variance was performed to evaluate the statistical significance of the difference among treatment groups. Differences between treatment groups at the specific time points were then assessed by using the Mann-Whitney test with Holm-Bonferroni's correction for multiple comparisons. A value of $p<0.05$ was considered statistically significant. Data analysis was performed with SPSS for Windows, version 10.0 (SPSS Inc., Chicago, IL, USA).

\section{RESULTS}

The potential role of human AM patches for the treatment of liver fibrosis was investigated in rats subjected to the common BDL and scission procedures, a classical experimental method to induce biliary fibrosis in animal models and to analyze the events leading from the initial injury of the bile ducts to the organization of a fibrotic reaction, and, finally, to the outcome of cirrhosis (21). The level of fibrosis achieved was firstly assessed by the classical semiquantitative Knodell fibrosis scoring system (7), which was applied to the histological exami- nation of rat liver tissues that had been subjected to Goldner's modified Masson trichrome staining. This method allows a staging of fibrotic and cirrhotic liver lesions, by considering not only the extent of ECM deposition (i.e., collagen deposition) but also the progressive expansion of the lesions from portal regions to gradually involving the interstitial parenchyma, and finally resulting in liver remodeling.

After BDL induction, the onset of the fibrotic injury is mainly characterized by periductular collagen deposits along the portal tracts (score 1). Subsequently, the fibrotic process infiltrates the hepatic parenchyma and forms bridging septa (linkage) between portal tracts and between portal tracts and the central vein (score 3). Finally, the liver architecture is severely compromised. A marked collagen accumulation is observed in the interstitial spaces with formation of fibrous septa separating and surrounding small nodules of parenchyma: this defines the cirrhosis stage (score 4). Representative photomicrographs of each fibrosis score, obtained at different times after the BDL procedure in the BDL rat group, are shown in Figure 1.

In both experimental groups (BDL and BDL $+\mathrm{AM}$ ), liver fibrosis became detectable 2 weeks post-BDL procedure and was located around biliary structures of the portal tracts. Interestingly, although in the control BDL group the fibrosis worsened progressively thereafter, reaching the cirrhosis stage at the last time point, in $\mathrm{BDL}+\mathrm{AM}$ rats the progression of liver fibrosis was less severe than that observed in BDL rats. Indeed, in BDL + $\mathrm{AM}$ rats, fibrosis remained confined at the portal/periportal areas, with no formation of complete fibrous septal linkage between portal tracts, no fibrotic process in the interstitial spaces of hepatic parenchyma and, importantly, no cirrhotic lesions were ever observed.

Semiquantitative Knodell scoring of these experimental results is reported in Figure 2. Specifically, at 4 weeks post-BDL, the median score values were 2.0 (IQR: 2.0) in BDL + AM rats and 3.0 (IQR: 1.0) in BDL rats $(\mathrm{p}<0.05)$. In contrast with BDL rats, which showed a continuous evolution of fibrosis from week 4 to week 6 [from 3.0 (IQR: 1.0) to 4.0 (IQR: 1.0) score units], $\mathrm{BDL}+\mathrm{AM}$ rats did not show any further fibrotic progression [the fibrosis median score value remained at 2.0 (IQR: 2.0) at both time points].

Considering that liver fibrotic injury induced by the BDL procedure is the outcome of a cascade of events and that multiple profibrogenic pathways are activated in this process, as well as undertaking the semiquantitative evaluation of the fibrotic process, we included in our study, as reported below, the quantitative assessment of three main parameters that are specifically involved in the establishment and progression of such a biliary 

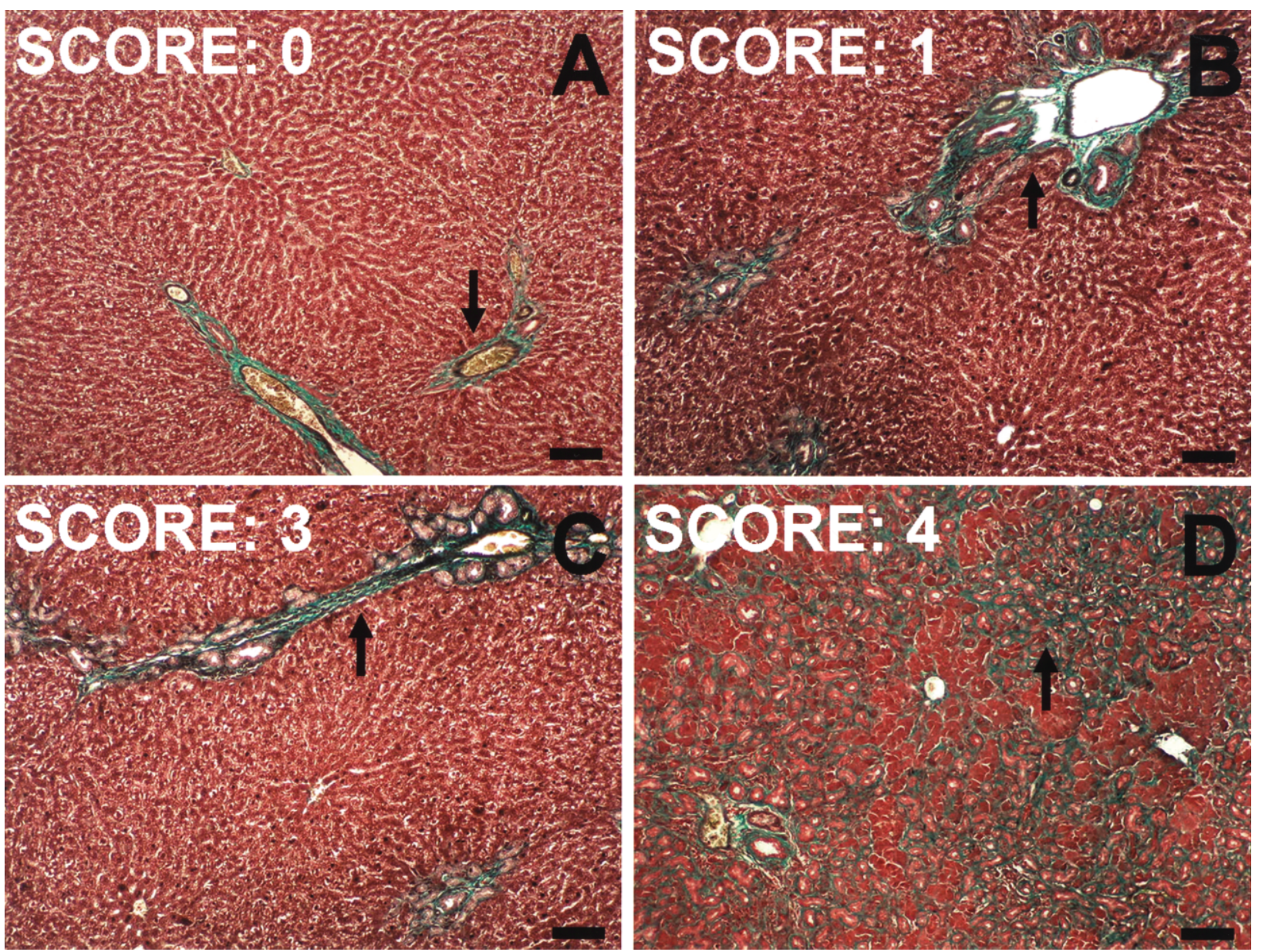

Figure 1. Sample pictures of semiquantitative Knodell scoring patterns for liver fibrosis. (A) Rat liver, score 0: the periportal area is morphologically normal and characterized by the presence of a thin rim of light green stained collagen (arrow). (B) Rat liver, score 1: portal tract is enlarged by the accumulation of periductular intensely green-stained collagen deposits (arrow). (C) Rat liver, score 3: collagen infiltration forms bridging septa between portal tracts (arrow). (D) Rat liver, score 4: collagen deposition infiltrates lobular parenchyma (arrow). Samples in (A-D): Goldner's modified Masson trichrome staining. Scale bar: $100 \mu \mathrm{m}$.

fibrotic lesion: ductular reaction, myofibroblast activation, and collagen deposition.

\section{Human AM Reduced BDL-Induced Ductular Reaction}

To evaluate the potential of human AM in reducing the ductular reaction (i.e., the abnormal proliferation of biliary structures) induced by the BDL procedure, we analyzed the expression of CK19, a marker of biliary epithelial cells, in the liver tissues of BDL and BDL + AM group rats.

In the liver tissues of the BDL group, the ductular reaction progressively and constantly increased from week 2 up to week 6 , as can be seen from representative photomicrographs, captured at different time points (Fig. $3 \mathrm{~A}-\mathrm{C})$. Specifically, at week 2, well-formed biliary structures were observed in the portal tracts and numer- ous bile ductules in the periportal areas (Fig. 3A). Four weeks after BDL, the ductular reaction continued in the portal/periportal areas and extended deeply into the hepatic lobule (Fig. 3B). At week 6, the ductular reaction was very intense and delineated complete nodules of hepatic parenchyma, with additional CK19-positive small ductules arising within these circumscribed nodules (Fig. 3C).

In BDL + AM rats, although the ductular reaction observed at week 2 was similar to that found in BDL rats, at the later time points it progressed more slowly than in BDL rats (Fig. 3D-F). Intriguingly, at week 6, in contrast to the BDL group, the ductular reaction did not circumscribe any nodules of hepatic parenchyma (Fig. 3F).

The areas occupied by CK19-positive cells were quantitatively evaluated by digital image analysis (Fig. 


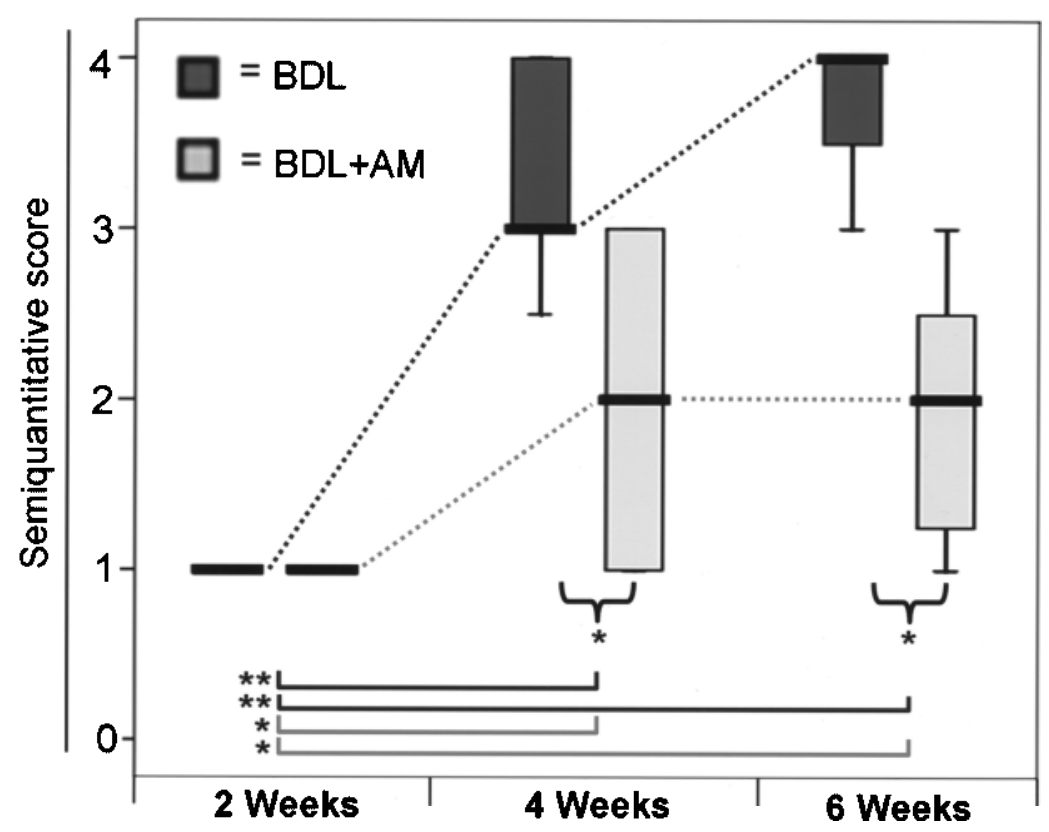

Figure 2. Semiquantitative Knodell scoring of experimental groups. Median with IQR range of 10 independent experiments is represented as boxplots for BDL (dark gray) and BDL + AM (light gray) groups, at each different time point. Dotted lines between boxplots represent the trend of median differences in BDL (dark gray) and BDL + AM (light gray) groups. Curly brackets represent significant difference between groups at the same time point. Lines at the base of boxplots represent significant difference between time points in BDL (dark gray) and BDL + AM (light gray) groups. $* p<0.05 ; * * p<0.01$.

3G-I). At week 2, in BDL rats CK19-positive cells occupied $5.36 \%$ (IQR: 3.40 ) of the total liver area (Fig. 3I). Subsequently, the area occupied by CK19-positive cells progressively increases. Indeed, at week 4 , this occupied $12.76 \%$ (IQR: 7.53 ) of the liver area (Fig. 3I) and at week 6 the hepatic parenchyma area involved was almost doubled to $18.82 \%$ (IQR: 9.75) (Fig. 3I).

In BDL + AM rats at week 4, only 5.91\% (IQR: 5.05) of the liver area was involved in ductular reaction $(p<$ 0.01 vs. BDL group, at the same time point) (Fig. 3I), and at week 6 it only weakly increased up to $9.45 \%$ (IQR: 3.85) ( $p<0.01$ vs. BDL group) (Fig. 3I).

\section{Human AM Reduced BDL-Induced Myofibroblast Activation}

Given that $\alpha$-SMA-positive and therefore activated myofibroblasts are the principal cells responsible for the collagen production and deposition observed in the fibrosis process (58), we assessed the potential of human AM to reduce myofibroblast activation by monitoring the presence of $\alpha$-SMA-positive cells in liver samples collected from rats of both experimental groups at different time points after BDL induction.

In normal liver, $\alpha$-SMA immunoreactivity is restricted to the wall of portal veins, hepatic arterioles, and terminal hepatic venules, whereas the bile ducts are $\alpha$ SMA negative (5).

In BDL rats, a progressive increase in $\alpha$-SMA immunoreactivity was found at all time points analyzed, and this colocalized with fibrosis (collagen deposition) around biliary structures. At week 2, $\alpha$-SMA-positive cells were found mainly in portal tracts (Fig. 4A). Afterwards, at week $4, \alpha$-SMA-positive cells surrounded the reactive bile ductules that formed portal-portal bridges during the progression of fibrosis (Fig. 4B) and at week $6, \alpha$-SMA immunoreactivity was also detected around ductules going deeply into the hepatic lobule and into perisinusoidal spaces (Fig. 4C).

$\alpha$-SMA immunoreactivity detected in rats treated with human AM was lower at all time points with respect to that found in BDL rats. In these rats, $\alpha$-SMApositive cells were found in the portal and periportal areas and, even at week 6 , they were confined to these areas without homogenous distribution around all biliary structures (i.e., not all periductular cells were immunopositive) (Fig. 4D-F).

The data obtained by the quantitative digital image analysis confirmed a lower presence of activated myofibroblasts in the livers of AM-treated rats with respect to BDL rats (Fig. 4G-I). Indeed, at week 2, the liver area 

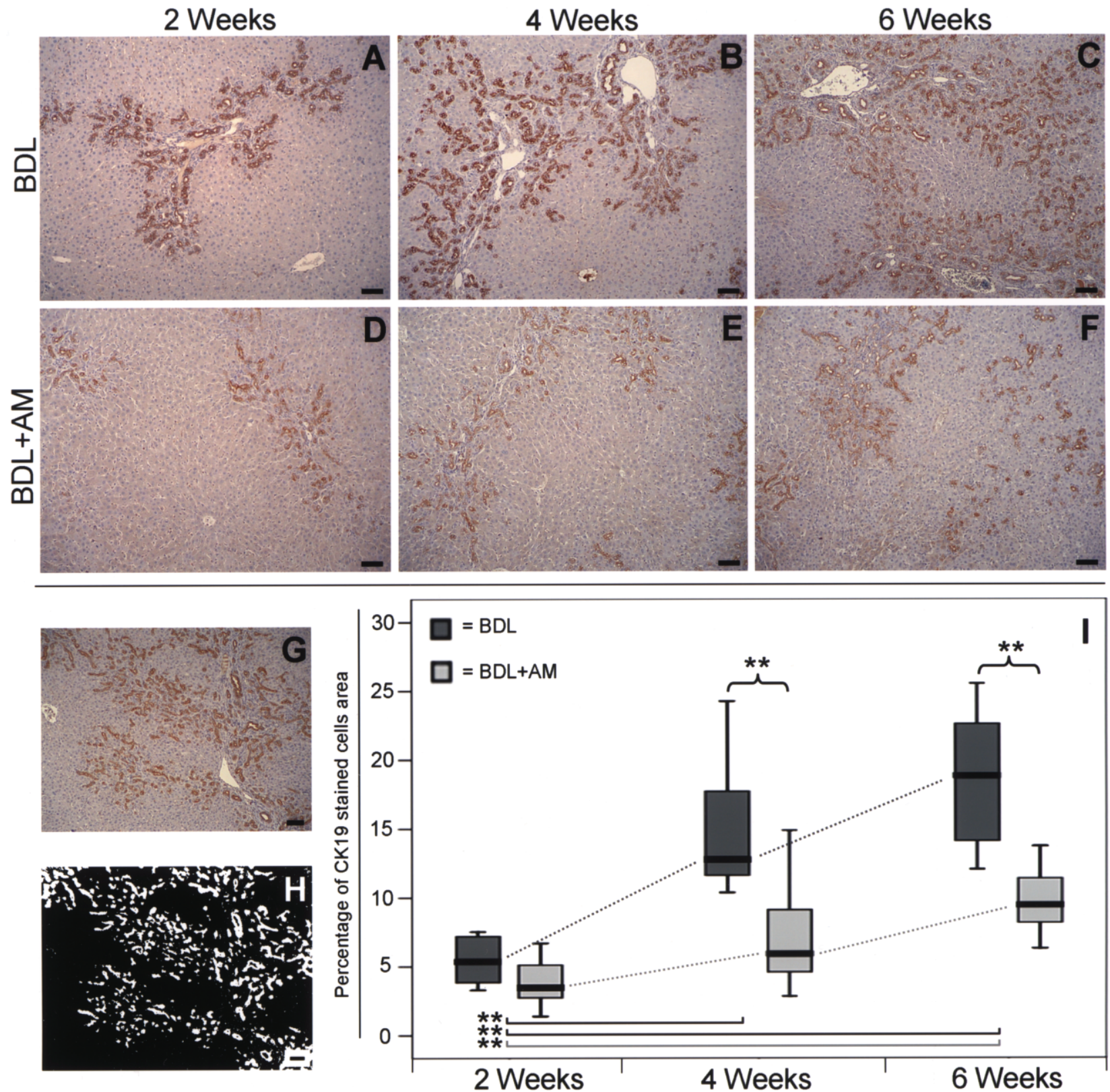

Figure 3. Quantitative evaluation of CK19 immunohistochemical staining in experimental groups. (A-C) Rat liver, BDL group: representative microphotographs at 2, 4, and 6 weeks, respectively. (D-F) Rat liver, BDL + AM group: representative microphotographs at 2, 4, and 6 weeks, respectively. (G) Sample microphotograph taken before the image analysis procedure used to extrapolate the CK19 staining from the image. (H) Sample image, same as in (G), representing a step of the image analysis procedure used: white areas are representative of CK19 staining. (I) Values of quantitative CK19 scoring in different groups: median with IQR range of 10 independent experiments is represented as boxplots for BDL (dark gray) and BDL + AM (light gray) groups, at each different time point. Dotted lines between boxplots represent the trend of median differences in BDL (dark gray) and BDL + AM (light gray) groups. Curly brackets represent significant difference between groups at the same time point. Lines at the bottom of boxplots represent significant difference between time points in BDL (dark gray) and BDL + AM (light gray) groups. * $p<0.05$; $* * p<0.01$. Samples in $(\mathrm{A}-\mathrm{G})$ were stained using indirect immunoperoxidase method with hematoxylin counterstaining. Scale bar: $100 \mu \mathrm{m}$. 

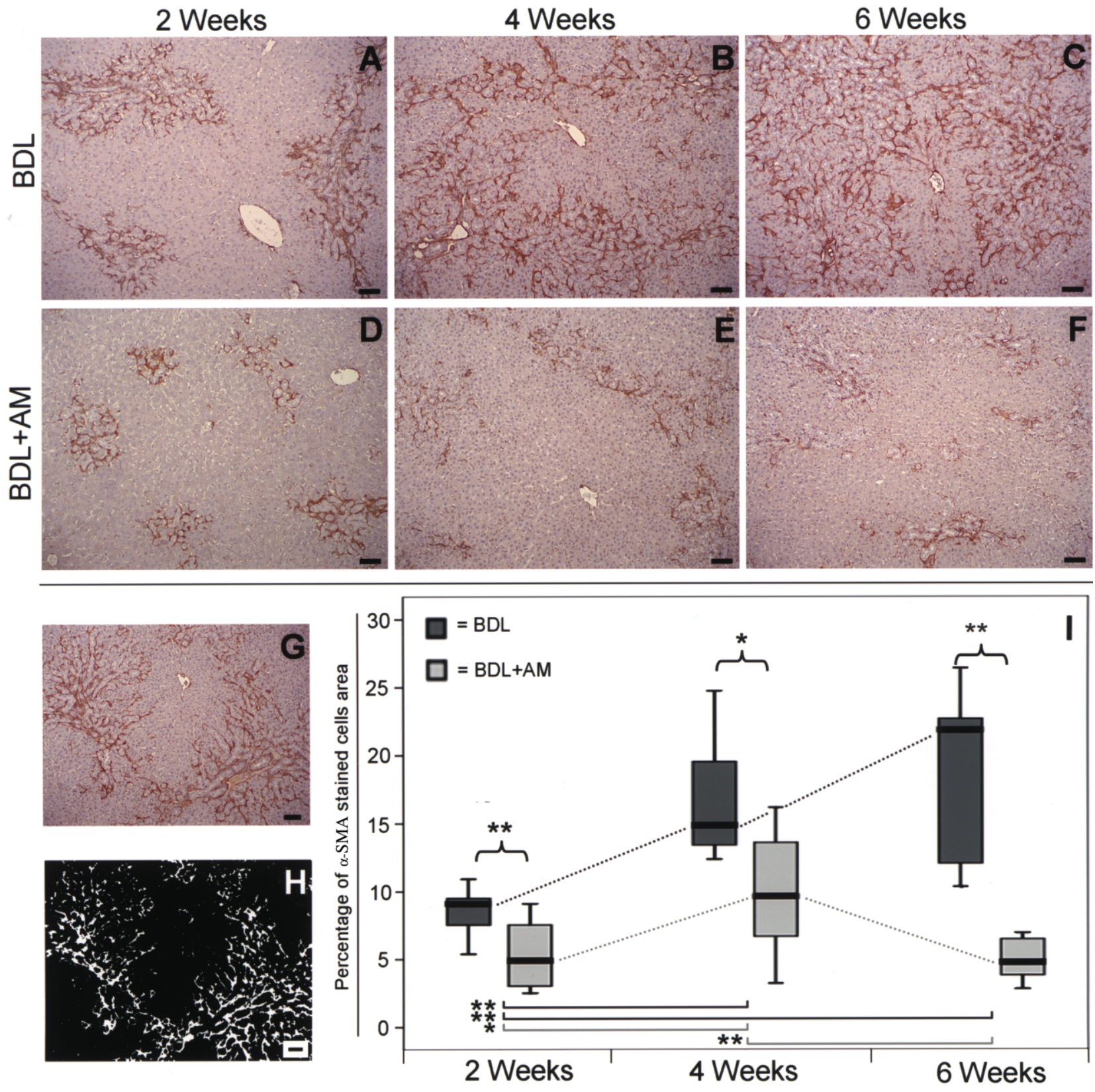

Figure 4. Quantitative evaluation of $\alpha$-SMA immunohistochemical staining in experimental groups. (A-C) Rat liver, BDL group: representative microphotographs at 2, 4, and 6 weeks, respectively. (D-F) Rat liver, BDL + AM group: representative microphotographs at 2, 4, and 6 weeks, respectively. (G) Sample microphotograph taken before the image analysis procedure used to extrapolate the $\alpha$-SMA staining from the image. $(\mathrm{H})$ Sample image, same as in $(\mathrm{G})$, representing a step of the image analysis procedure used: white areas are representative of $\alpha$-SMA staining. (I) Values of quantitative $\alpha$-SMA scoring in different groups: Median with IQR range of 10 independent experiments is represented as boxplots for BDL (dark gray) and BDL + AM (light gray) groups, at each different time point. Dotted lines between boxplots represent the trend of median differences in BDL (dark gray) and BDL + AM (light gray) groups. Curly brackets represent significant difference between groups at the same time point. Lines at the bottom of boxplots represent significant difference between time points in BDL (dark gray) and BDL + AM (light gray) groups. $* p<0.05$; $* * p<0.01$. Samples in $(\mathrm{A}-\mathrm{G})$ were stained using indirect immunoperoxidase method with hematoxylin counterstaining. Scale bar: $100 \mu \mathrm{m}$. 
occupied by $\alpha$-SMA-positive cells in the AM-treated rats was only $4.90 \%$ (IQR: 4.62) while in BDL rats it was $9.11 \%$ (IQR: 3.39$)(p<0.01$ vs. BDL group at the same time point) (Fig. 4I). At week 4, $\alpha$-SMA-positive cells occupied $9.63 \%$ of the liver area (IQR: 7.72 ) in $\mathrm{BDL}+\mathrm{AM}$ rats $(p<0.05$ vs. BDL group) and $14.87 \%$ (IQR: 6.92) in BDL rats (Fig. 4I). Intriguingly, at week 6 , while in BDL group the liver area occupied by the activated myofibroblasts increased to $21.85 \%$ (IQR: 11.10) (Fig. 4I), in the AM-treated rats it was $4.76 \%$ (IQR: 3.10$)$ ( $p<0.01$ vs. BDL group), an area even smaller than that occupied at week 4 and similar to that occupied at week 2 (Fig. 4I).

\section{Human AM Decreased the BDL-Induced Collagen Deposition}

To quantitatively evaluate the effects of human AM on liver collagen deposition, Goldner's modified Masson trichrome staining-positive areas were analyzed and quantified by image analysis morphometry in liver samples collected from rats of both experimental groups. Representative photomicrographs and quantitative data collected after 2, 4, and 6 weeks from BDL and BDL + $\mathrm{AM}$ rats are shown in Figure 5.

In BDL rats, from week 2 to week 4, liver collagen deposition markedly increased and no further significant expansion of occupied liver area was observed after week 4 (Fig. 5A-C). Furthermore, although collagen deposition observed at week 2 was similar in the two group of animals, afterwards no further progression was observed in the BDL + AM rats (Fig. 5D-F).

The quantitative evaluation by digital image analysis confirmed that, at week 2, collagen deposition was comparable in rats of both groups (Fig. 5G-I) but subsequently it was significantly reduced in the AM-treated rats. Indeed, in BDL rats at week 4, the collagen deposition area involved $20.61 \%$ (IQR: 6.78) of the liver area (Fig. 5I) and at week 6, it remained at $18.38 \%$ (IQR: 8.29) (Fig. 5I). In contrast, in BDL + AM rats, liver areas occupied by collagen at week 4 and week 6 were only $9.92 \%$ (IQR: 2.15 ) and $11.35 \%$ (IQR: 1.54), respectively $(p<0.01$ vs. BDL group at the same time points) (Fig. 5I).

\section{DISCUSSION}

This study demonstrates that human AM, when applied as a patch onto the liver surface, reduces progression of experimental biliary fibrosis induced in rats by the BDL procedure. Combining a semiquantitative evaluation of the fibrotic process and the quantitative assessment of three main parameters specifically involved in the establishment and progression of biliary fibrosis (i.e., ductular reaction, myofibroblast activation, and collagen deposition), our findings suggest that human
AM could serve as a hepatoprotective agent against hepatic damage associated with fibrotic degeneration.

Biliary fibrosis and resultant cirrhosis are among the most common outcomes of chronic liver diseases. Unfortunately, the therapeutic repertoire currently available for treating liver fibrosis and cirrhosis of this type and others is very limited and, often, the only effective remaining treatment is orthotopic liver transplantation $(1,36)$. Therefore, strategies to reduce hepatic fibrosis and to avoid the need for organ transplantation are strongly required. To our knowledge, this is the first study aiming at investigating whether human AM patches can be used as a possible treatment for biliary fibrosis in an animal model (BDL-treated rat) commonly used for the study of such a type of fibrosis, and that mimics very well the situation of extrahepatic cholestasis arising in a variety of human liver diseases (21, 30,40).

First, we have demonstrated that human AM application reduces liver fibrosis when evaluated using the classical semiquantitative Knodell scoring system. Indeed, while in the BDL rat group, fibrosis showed a rapid progression leading to cirrhosis within 6 weeks, with collagen accumulation around ductular structures and in the interstitial spaces, in the BDL + AM rat group the application of human AM was able to confine the fibrosis to the portal/periportal area, with no formation of complete fibrous septal linkage between portal tracts and no further progression in liver fibrosis after 4 weeks from BDL.

By conducting an in-depth analysis of the stages that characterize biliary fibrosis induced by the BDL procedure, our results indicate that the application of human AM inhibits the cascade of events leading to liver fibrosis/cirrhosis in such a model, resulting in a final reduction of hepatic fibrosis.

Specifically, we have shown that the application of human AM slows the gradual progression of the ductular reaction, to the point that it is significantly reduced within 6 weeks in BDL + AM rats compared to the BDL rat group. This is a very important finding, considering that the ductular reaction (i.e., cholangiocyte proliferation and increased number of ductules) is one of the first process triggered by the BDL procedure and is thought to have a key role in the initiation and progression of liver fibrosis $(13,21,41)$. These results are in line with those reported by Chen et al. (10). Indeed, these authors, using the BDL model to induce liver fibrosis, demonstrated that chromium attenuation of liver fibrosis was first evidenced by a reduction in the severity of BDLinduced ductular reaction.

Following ductular proliferation, the chronic obstruction of bile duct causes massive activation of myofibroblasts in the periductal region and at the end results 

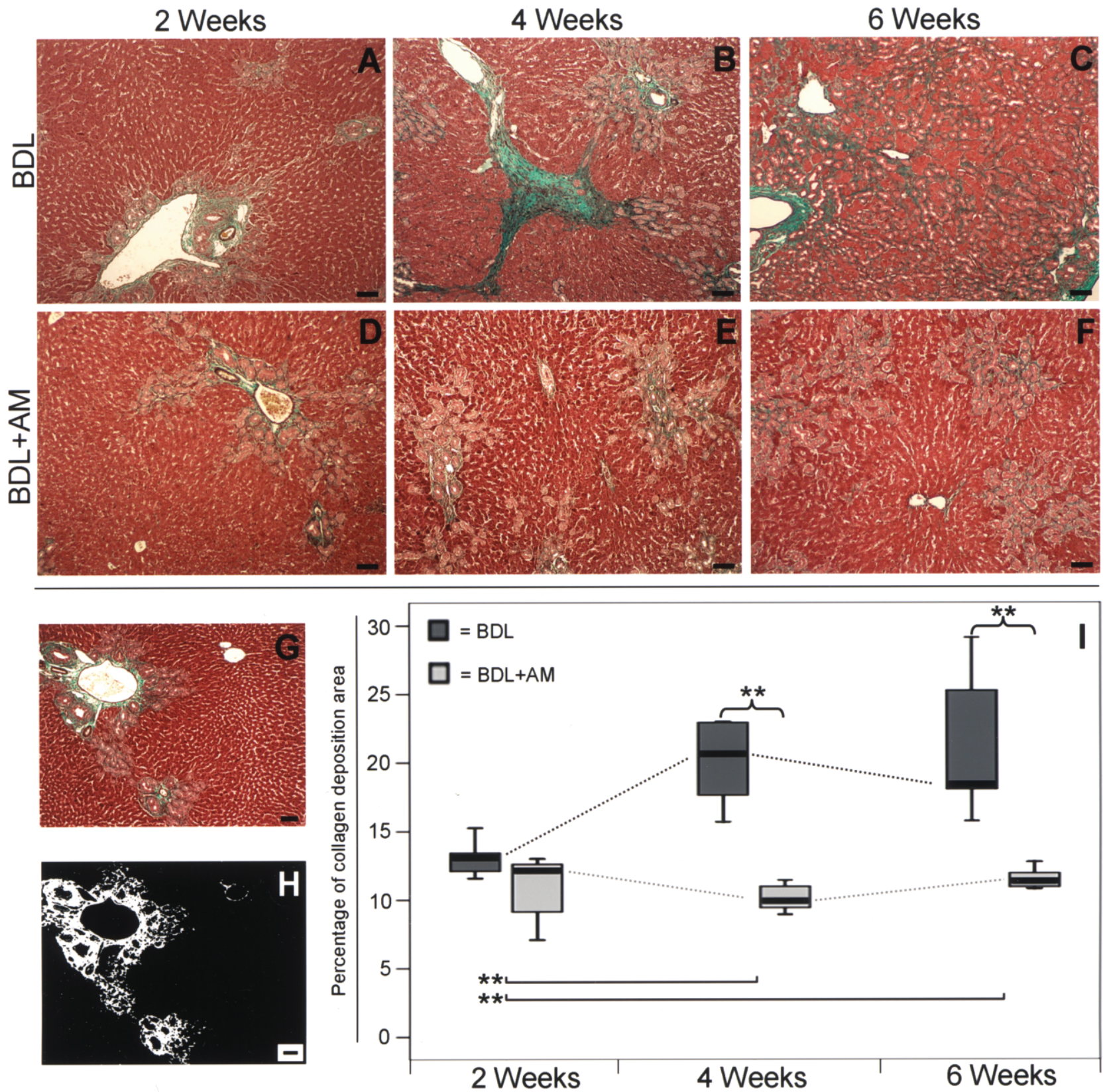

Figure 5. Quantitative evaluation of collagen histochemical staining in experimental groups. (A-C) Rat liver, BDL group: representative microphotographs at 2, 4, and 6 weeks, respectively. (D-F) Rat liver, BDL + AM group: representative microphotographs at 2, 4, and 6 weeks, respectively. (G) Sample microphotograph taken before the image analysis procedure used to extrapolate the collagen staining from the image. $(\mathrm{H})$ Sample image, same as in $(\mathrm{G})$, representing a step of the image analysis procedure used: white areas are representative of green collagen staining. (I) Values of quantitative collagen deposition in different groups: Median with IQR range of 10 independent experiments is represented as boxplots for BDL (dark gray) and BDL + AM (light gray) groups, at each different time point. Dotted lines between boxplots represent the trend of median differences in BDL (dark gray) and BDL + AM (light gray) groups. Curly brackets represent significant difference between groups at the same time point. Lines at the bottom of boxplots represent significant difference between time points in BDL (dark gray) and BDL + AM (light gray) groups. $* p<0.05 ; * * p<0.01$. Samples in $(\mathrm{A}-\mathrm{G})$ were stained using Goldner's modified Masson trichrome staining procedure. Scale bar: $100 \mu \mathrm{m}$. 
in biliary fibrosis/cirrhosis $(3,59)$. From this study we found that human AM reduces the occurrence of $\alpha$ SMA-expressing cells in the hepatic parenchyma after BDL induction, and this was already evident 2 weeks after BDL. $\alpha$-SMA is the histological hallmark of activated myofibroblasts, which secrete large amounts of ECM proteins, including collagen, and increase ECM accumulation during fibrosis (58). Given that, in biliary fibrosis induced by BDL, $\alpha$-SMA-positive myofibroblasts may originate not only from hepatic stellate cells (HSCs)/residential fibroblasts, but also from the bile duct epithelium through epithelial-mesenchymal transition (EMT) $(21,59)$, the reduction in the area occupied by myofibroblasts could be correlated, at least in part, with the decreased ductular reaction area observed in the rat group treated with AM.

Finally, our data suggest that the reduction in ductular proliferation and in myofibroblast activation could participate in the attenuation of the collagen deposition process. Although at 2 weeks after BDL the collagen deposition area was not significantly different in BDL + $\mathrm{AM}$ rats compared to the BDL rats, after 4 and 6 weeks, collagen deposition was reduced by about $50 \%$ in the $\mathrm{BDL}+\mathrm{AM}$ rats group. These data indicate a novel strategy for reducing collagen deposition in a BDL model of biliary fibrosis. Indeed, other studies reported to date using this same experimental model have demonstrated reduction of hepatic collagen deposition by means of other therapeutic agents, such as chromium supplementation (10) and hepatocyte growth factor $(14,59)$.

Taken together, these data indicate that the human $\mathrm{AM}$ does not prevent the formation of fibrosis, but certainly inhibits its progression to the end stage of disease.

The exact mechanism whereby human AM exerts these beneficial effects on BDL-induced liver fibrosis remains to be defined, and at present we can only speculate as to the nature of such mechanisms. Because by in situ hybridization analysis (for human genome-specific ALU-repetitive DNA sequences and human chromosome 17 centromere) (data not shown) no human cells were detected in the sections of AM-treated rat livers, this suggests that, within the limits of these experimental methods, no massive (or at least very low/undetectable) engraftment of AM-derived cells occurred in the host liver. Although we cannot exclude the possibility that few AM-derived cells engrafted in the liver, it is conceivable that the beneficial effects exerted by AM patch application might not be due to a replacement mechanism, in which engrafted AM-derived cells differentiated toward the hepatogenic lineage, but that, most likely, the effects observed were associated with the release of soluble factors by cells of the human AM patch (and of the few engrafted cells), which exerted paracrine effects. This mechanism, based on paracrine effects, has also been hypothesized by Tsai et al. (55) to explain the observed reduction in liver fibrosis in the absence of differentiation of engrafted Wharton's jelly-derived cells, which had been transplanted into rats with carbon tetrachloride $\left(\mathrm{CCl}_{4}\right)$-induced liver fibrosis. In addition, we have recently hypothesized such a paracrine mechanism to explain the ability of placenta-derived cells to reduce bleomycin-induced lung fibrosis (9) and of human AM patches to exert a protective effect against ischemia-induced changes in cardiac functions and dimensions (8). This hypothesis stems from the fact that the AM, as well as cells derived from this tissue, are able to release several factors that have potent immunomodulatory, anti-inflammatory, and antiscarring effects $(12,50)$.

Nevertheless, the identification of the specific paracrine bioactive molecules produced by AM and their target cells involved in the antifibrotic effects of AM on the liver remains to be explored. Considering that several studies have suggested that proliferating cholangiocytes have a role in the induction of liver biliary fibrosis, either directly, via EMT and therefore providing a source of fibrogenic myofibroblasts, or indirectly via activation of other liver cell types, such as resident portal fibroblasts and HSCs (21), by means of autocrine and paracrine mediators, $(21,26,59)$, it is conceivable that one of the targets of the paracrine actions of human AM might be cholangiocytes. Furthermore, taking into account the fact that the AM can modulate TGF- $\beta 1$ synthesis and/or signaling pathways $(29,47,56)$, it is possible that the human AM patch might lead to a reduction in the expression of profibrogenic factors such as TGF- $\beta 1$ and PDGF, or proinflammatory cytokines (e.g., IL-1, IL-6, etc.). To perform an exhaustive assessment of fibrosis and avoid potential confusing findings deriving from incomplete sampling, our approach called for whole organ resection and formalin fixation, which prevented us from performing expression studies in the livers of our experimental animals. However, it is worth noting that reduction of TGF- $\beta 1$ expression has been previously suggested to explain part of the mechanism of fibrosis reduction achieved by other approaches, such as MSC transplantation $(25,55)$, HGF administration (59), and chromium supplementation (10).

Nonetheless, direct testing of the potential paracrine function of the AM, as well as of other cell types that have been shown to have anti-inflammatory and antifibrotic effects $(11,55)$, would add mechanistic insights to our findings and clearly warrants further experimentations.

Because the same therapeutic approach may exert different, and even opposite, effects (reduction versus worsening) on fibrogenesis according to the type of fibrosis/cirrhosis (57), the beneficial effects achieved with the human AM patch on BDL-induced biliary fibrosis 
remain to be proven for other types of fibrosis. However, considering that the results obtained to date using stem cell transplantation are often conflicting $(1,2,11)$, and in many cases their successes have been related to the level of engraftment in the liver and differentiation achieved $(1,11,36)$, a strategy like the one we used, that seems not depend on the level of cell engraftment achieved, might be very beneficial and innovative.

In conclusion, the results of our study strengthen previous indication on the antifibrotic effect of AM and suggest that human AM patching might inhibit fibrosis progression in BDL-injured livers. Even though the mechanisms of this antifibrotic effect remain an unexplored issue, but certainly of crucial importance, and it also remains to be clarified as to whether the beneficial effects of AM can also be conferred in disease models where fibrosis is already established, our results open the way for further investigations aimed at extending the potential use of the human AM for liver fibrotic diseases.

ACKNOWLEDGMENTS: We thank the Department of Obstetrics and Gynecology of Fondazione Poliambulanza Istituto Ospedaliero, Brescia, Italy. We also thank Dr. Marco Evangelista for help in editing the manuscript and Dr. Nilson Sant'Anna for help in setting up the image analysis system. This study was supported by grants from Fondazione Cariplo (Rif. 2006.0762/11.6457). Dr. Luciana Barros Sant'Anna was supported by Coordenação de Aperfeiçoamento de Pessoal de Nivel Superior (CAPES), grant No. 3813/08-8.

\section{REFERENCES}

1. Alison, M. R.; Islam, S.; Lim, S. Stem cells in liver regeneration, fibrosis and cancer: The good, the bad and the ugly. J. Pathol. 217:282-298; 2009.

2. Alison, M. R.; Islam, S.; Lim, S. M. Cell therapy for liver disease. Curr. Opin. Mol. Ther. 11:364-374; 2009.

3. Aller, M. A.; Arias, J. L.; Garcia-Dominguez, J.; Arias, J. I.; Duran, M.; Arias, J. Experimental obstructive cholestasis: The wound-like inflammatory liver response. Fibrogenesis Tissue Repair 1:6-22; 2008.

4. Bailo, M.; Soncini, M.; Vertua, E.; Signoroni, P. B.; Sanzone, S.; Lombardi, G.; Arienti, D.; Calamani, F.; Zatti, D.; Paul, P.; Albertini, A.; Zorzi, F.; Cavagnini, A.; Candotti, F.; Wengler, G. S.; Parolini, O. Engraftment potential of human amnion and chorion cells derived from term placenta. Transplantation 78:1439-1448; 2004

5. Beaussier, M.; Wendum, D.; Schiffer, E.; Dumont, S.; Rey, C.; Lienhart, A.; Housset, C. Prominent contribution of portal mesenchymal cells to liver fibrosis in ischemic and obstructive cholestatic injuries. Lab. Invest. 87:292303; 2007.

6. Beck, P. L.; Lee, S. S. Vitamin K1 improves survival in bile-duct-ligated rats with cirrhosis. J. Hepatol. 23:235; 1995.

7. Brunt, E. M. Grading and staging the histopathological lesions of chronic hepatitis: The Knodell histology activity index and beyond. Hepatology 31:241-246; 2000.

8. Cargnoni, A.; Di Marcello, M.; Campagnol, M.; Nassuato, C.; Albertini, A.; Parolini, O. Amniotic membrane patching promotes ischemic rat heart repair. Cell Transplant. 18:1147-1159; 2009.
9. Cargnoni, A.; Gibelli, L.; Tosini, A.; Signoroni, P. B.; Nassuato, C.; Arienti, D.; Lombardi, G.; Albertini, A.; Wengler, G. S.; Parolini, O. Transplantation of allogeneic and xenogeneic placenta-derived cells reduces bleomycininduced lung fibrosis. Cell Transplant. 18:405-422; 2009.

10. Chen, W. Y.; Chen, C. J.; Liao, J. W.; Mao, F. C. Chromium attenuates hepatic damage in a rat model of chronic cholestasis. Life Sci. 84:606-614; 2009.

11. Dai, L. J.; Li, H. Y.; Guan, L. X.; Ritchie, G.; Zhou, J. X. The therapeutic potential of bone marrow-derived mesenchymal stem cells on hepatic cirrhosis. Stem Cell Res. 2: $16-25 ; 2009$.

12. Denison, F. C.; Kelly, R. W.; Calder, A. A.; Riley, S. C. Cytokine secretion by human fetal membranes, decidua and placenta at term. Hum. Reprod. 13:3560-3565; 1998.

13. Desmet, V.; Roskams, T.; Van Eyken, P. Ductular reaction in the liver. Pathol. Res. Pract. 191:513-524; 1995.

14. Diaz-Gil, J. J.; Garcia-Monzon, C.; Rua, C.; Martin-Sanz, P.; Cereceda, R. M.; Miquilena-Colina, M. E.; Machin, C.; Fernandez-Martinez, A.; Garcia-Canero, R. Liver growth factor antifibrotic activity in vivo is associated with a decrease in activation of hepatic stellate cells. Histol. Histopathol. 24:473-479; 2009.

15. Dua, H. S.; Gomes, J. A.; King, A. J.; Maharajan, V. S. The amniotic membrane in ophthalmology. Surv. Ophthalmol. 49:51-77; 2004.

16. Eichna, D. M.; Brown, K. S.; Breen, A.; Dean, R. B. Mucormycosis: A rare but serious infection. Clin. J. Oncol. Nurs. 12:108-112; 2008.

17. Evangelista, M.; Soncini, M.; Parolini, O. Placentaderived stem cells: New hope for cell therapy? Cytotechnology 58:33-42; 2008.

18. Fernandes, M.; Sridhar, M. S.; Sangwan, V. S.; Rao, G. N. Amniotic membrane transplantation for ocular surface reconstruction. Cornea 24:643-653; 2005.

19. Fisher, R. A.; Strom, S. C. Human hepatocyte transplantation: Worldwide results. Transplantation 82:441-449; 2006.

20. Fitzpatrick, E.; Mitry, R. R.; Dhawan, A. Human hepatocyte transplantation: State of the art. J. Intern. Med. 266: 339-357; 2009.

21. Glaser, S. S.; Gaudio, E.; Miller, T.; Alvaro, D.; Alpini, G. Cholangiocyte proliferation and liver fibrosis. Expert Rev. Mol. Med. 11:e7; 2009.

22. Gomes, J. A.; Romano, A.; Santos, M. S.; Dua, H. S. Amniotic membrane use in ophthalmology. Curr. Opin. Ophthalmol. 16:233-240; 2005.

23. Henderson, N. C.; Forbes, S. J. Hepatic fibrogenesis: From within and outwith. Toxicology 254:130-135; 2008.

24. Hennerbichler, S.; Reichl, B.; Pleiner, D.; Gabriel, C.; Eibl, J.; Redl, H. The influence of various storage conditions on cell viability in amniotic membrane. Cell Tissue Bank 8:1-8; 2007.

25. Jung, K. H.; Shin, H. P.; Lee, S.; Lim, Y. J.; Hwang, S. H.; Han, H.; Park, H. K.; Chung, J. H.; Yim, S. V. Effect of human umbilical cord blood-derived mesenchymal stem cells in a cirrhotic rat model. Liver Int. 29:898909; 2009 .

26. Kinnman, N.; Francoz, C.; Barbu, V.; Wendum, D.; Rey, C.; Hultcrantz, R.; Poupon, R.; Housset, C. The myofibroblastic conversion of peribiliary fibrogenic cells distinct from hepatic stellate cells is stimulated by plateletderived growth factor during liver fibrogenesis. Lab. Invest. 83:163-173; 2003.

27. Lamprecht, M. R.; Sabatini, D. M.; Carpenter, A. E. CellProfiler: Free, versatile software for automated biological image analysis. Biotechniques 42:71-75; 2007. 
28. Laurson, J.; Selden, C.; Hodgson, H. J. Hepatocyte progenitors in man and in rodents-multiple pathways, multiple candidates. Int. J. Exp. Pathol. 86:1-18; 2005.

29. Lee, S. B.; Li, D. Q.; Tan, D. T.; Meller, D. C.; Tseng, S. C. Suppression of TGF-beta signaling in both normal conjunctival fibroblasts and pterygial body fibroblasts by amniotic membrane. Curr. Eye Res. 20:325-334; 2000.

30. Li, M. K.; Crawford, J. M. The pathology of cholestasis. Semin. Liver Dis. 24:21-42; 2004.

31. Llovet, J. M.; Schwartz, M.; Mazzaferro, V. Resection and liver transplantation for hepatocellular carcinoma. Semin. Liver Dis. 25:181-200; 2005.

32. Lysy, P. A.; Campard, D.; Smets, F.; Najimi, M.; Sokal, E. M. Stem cells for liver tissue repair: Current knowledge and perspectives. World J. Gastroenterol. 14:864-875; 2008.

33. Miki, T.; Lehmann, T.; Cai, H.; Stolz, D. B.; Strom, S. C. Stem cell characteristics of amniotic epithelial cells. Stem Cells 23:1549-1559; 2005.

34. Miki, T.; Strom, S. C. Amnion-derived pluripotent/multipotent stem cells. Stem Cell Rev. 2:133-142; 2006.

35. Mukherjee, S.; Sorrell, M. F. Controversies in liver transplantation for hepatitis C. Gastroenterology 134:1777$1788 ; 2008$.

36. Nussler, A.; Konig, S.; Ott, M.; Sokal, E.; Christ, B.; Thasler, W.; Brulport, M.; Gabelein, G.; Schormann, W.; Schulze, M.; Ellis, E.; Kraemer, M.; Nocken, F.; Fleig, W.; Manns, M.; Strom, S. C.; Hengstler, J. G. Present status and perspectives of cell-based therapies for liver diseases. J. Hepatol. 45:144-159; 2006.

37. Parolini, O.; Alviano, F.; Bagnara, G. P.; Bilic, G.; Buhring, H. J.; Evangelista, M.; Hennerbichler, S.; Liu, B.; Magatti, M.; Mao, N.; Miki, T.; Marongiu, F.; Nakajima, H.; Nikaido, T.; Portmann-Lanz, C. B.; Sankar, V.; Soncini, M.; Stadler, G.; Surbek, D.; Takahashi, T. A.; Redl, H.; Sakuragawa, N.; Wolbank, S.; Zeisberger, S.; Zisch, A.; Strom, S. C. Concise review: Isolation and characterization of cells from human term placenta: Outcome of the first international Workshop on Placenta Derived Stem Cells. Stem Cells 26:300-311; 2008.

38. Pinzani, M.; Rombouts, K. Liver fibrosis: From the bench to clinical targets. Dig. Liver Dis. 36:231-242; 2004.

39. Portmann-Lanz, C. B.; Schoeberlein, A.; Huber, A.; Sager, R.; Malek, A.; Holzgreve, W.; Surbek, D. V. Placental mesenchymal stem cells as potential autologous graft for pre- and perinatal neuroregeneration. Am. J. Obstet. Gynecol. 194:664-673; 2006.

40. Rodriguez-Garay, E. A. Cholestasis: Human disease and experimental animal models. Ann. Hepatol. 2:150-158 2003.

41. Roskams, T.; Desmet, V. Ductular reaction and its diagnostic significance. Semin. Diagn. Pathol. 15:259-269; 1998.

42. Roskams, T. A.; Theise, N. D.; Balabaud, C.; Bhagat, G.; Bhathal, P. S.; Bioulac-Sage, P.; Brunt, E. M.; Crawford, J. M.; Crosby, H. A.; Desmet, V.; Finegold, M. J.; Geller, S. A.; Gouw, A. S.; Hytiroglou, P.; Knisely, A. S.; Kojiro, M.; Lefkowitch, J. H.; Nakanuma, Y.; Olynyk, J. K.; Park, Y. N.; Portmann, B.; Saxena, R.; Scheuer, P. J.; Strain, A. J.; Thung, S. N.; Wanless, I. R.; West, A. B. Nomenclature of the finer branches of the biliary tree: canals, ductules, and ductular reactions in human livers. Hepatology 39:1739-1745; 2004.

43. Said, A.; Lucey, M. R. Liver transplantation: An update 2008. Curr. Opin. Gastroenterol. 24:339-345; 2008.
44. Sancho-Bru, P.; Najimi, M.; Caruso, M.; Pauwelyn, K.; Cantz, T.; Forbes, S.; Roskams, T.; Ott, M.; Gehling, U.; Sokal, E.; Verfaillie, C. M.; Muraca, M. Stem and progenitor cells for liver repopulation: Can we standardise the process from bench to bedside? Gut 58:594-603; 2009.

45. Sato, Y.; Harada, K.; Ozaki, S.; Furubo, S.; Kizawa, K.; Sanzen, T.; Yasoshima, M.; Ikeda, H.; Sasaki, M.; Nakanuma, Y. Cholangiocytes with mesenchymal features contribute to progressive hepatic fibrosis of the polycystic kidney rat. Am. J. Pathol. 171:1859-1871; 2007.

46. Schutte, K.; Bornschein, J.; Malfertheiner, P. Hepatocellular carcinoma-epidemiological trends and risk factors. Dig. Dis. 27:80-92; 2009.

47. Shek, F. W.; Benyon, R. C. How can transforming growth factor beta be targeted usefully to combat liver fibrosis? Eur. J. Gastroenterol. Hepatol. 16:123-126; 2004.

48. Skalli, O.; Ropraz, P.; Trzeciak, A.; Benzonana, G.; Gillessen, D.; Gabbiani, G. A monoclonal antibody against alpha-smooth muscle actin: A new probe for smooth muscle differentiation. J. Cell Biol. 103:2787-2796; 1986.

49. Soncini, M.; Vertua, E.; Gibelli, L.; Zorzi, F.; Denegri, M.; Albertini, A.; Wengler, G. S.; Parolini, O. Isolation and characterization of mesenchymal cells from human fetal membranes. J. Tissue Eng. Regen. Med. 1:296-305; 2007.

50. Steed, D. L.; Trumpower, C.; Duffy, D.; Smith, C.; Marshall, V.; Rupp, R.; Robson, M. Amnion-derived cellular cytokine solution: A physiological combination of cytokines for wound healing. Eplasty 8:e18; 2008.

51. Strom, S. C.; Bruzzone, P.; Cai, H.; Ellis, E.; Lehmann, T.; Mitamura, K.; Miki, T. Hepatocyte transplantation: Clinical experience and potential for future use. Cell Transplant. 15(Suppl. 1):S105-110; 2006.

52. Takashima, S.; Ise, H.; Zhao, P.; Akaike, T.; Nikaido, T Human amniotic epithelial cells possess hepatocyte-like characteristics and functions. Cell Struct. Funct. 29:73$84 ; 2004$

53. Tamagawa, T.; Ishiwata, I.; Saito, S. Establishment and characterization of a pluripotent stem cell line derived from human amniotic membranes and initiation of germ layers in vitro. Hum. Cell 17:125-130; 2004.

54. Tamagawa, T.; Oi, S.; Ishiwata, I.; Ishikawa, H.; Nakamura, Y. Differentiation of mesenchymal cells derived from human amniotic membranes into hepatocyte-like cells in vitro. Hum. Cell 20:77-84; 2007.

55. Tsai, P. C.; Fu, T. W.; Chen, Y. M.; Ko, T. L.; Chen, T. H.; Shih, Y. H.; Hung, S. C.; Fu, Y. S. The therapeutic potential of human umbilical mesenchymal stem cells from Wharton's jelly in the treatment of rat liver fibrosis. Liver Transpl. 15:484-495; 2009.

56. Tseng, S. C.; Li, D. Q.; Ma, X. Suppression of transforming growth factor-beta isoforms, TGF-beta receptor type II, and myofibroblast differentiation in cultured human corneal and limbal fibroblasts by amniotic membrane matrix. J. Cell. Physiol. 179:325-335; 1999.

57. Van de Casteele, M.; Roskams, T.; Van der Elst, I.; van Pelt, J. F.; Fevery, J.; Nevens, F. Halofuginone can worsen liver fibrosis in bile duct obstructed rats. Liver Int. 24:502-509; 2004.

58. Wynn, T. A. Common and unique mechanisms regulate fibrosis in various fibroproliferative diseases. J. Clin. Invest. 117:524-529; 2007

59. Xia, J. L.; Dai, C.; Michalopoulos, G. K.; Liu, Y. Hepatocyte growth factor attenuates liver fibrosis induced by bile duct ligation. Am. J. Pathol. 168:1500-1512; 2006. 
